Binghamton University

The Open Repository @ Binghamton (The ORB)

\title{
Experimental characterization of the electrostatic levitation force in MEMS transducers
}

Meysam Daeichin

Binghamton University--SUNY, mdaeich1@binghamton.edu

Ronald Miles

Binghamton University--SUNY, rmiles@binghamton.edu

Shahrzad Towfighian

Binghamton University--SUNY, stowfigh@binghamton.edu

Follow this and additional works at: https://orb.binghamton.edu/mechanical_fac

Part of the Mechanical Engineering Commons

\section{Recommended Citation}

Daeichin, Meysam; Miles, Ronald; and Towfighian, Shahrzad, "Experimental characterization of the electrostatic levitation force in MEMS transducers" (2020). Mechanical Engineering Faculty Scholarship. 29.

https://orb.binghamton.edu/mechanical_fac/29

This Article is brought to you for free and open access by the Mechanical Engineering at The Open Repository @ Binghamton (The ORB). It has been accepted for inclusion in Mechanical Engineering Faculty Scholarship by an authorized administrator of The Open Repository @ Binghamton (The ORB). For more information, please contact ORB@binghamton.edu. 


\title{
Experimental characterization of the electrostatic levitation force in MEMS transducers
}

\author{
Meysam Daeichin, Ronald N. Miles and Shahrzad Towfighian
}

\begin{abstract}
In this study, a two-step experimental procedure is described to determine the electrostatic levitation force in MEMS transducers. In these two steps, the microstructure is excited quasi-statically and dynamically and its response is used to derive the electrostatic force. The experimental results are obtained for a 1 by 1 plate that employs 112 levitation units. The experimentally obtained force is used in a lumped parameter model to find the microstructure response when it is subjected to different dynamical loads. The natural frequency and the damping ratios in the model are identified from the experimental results. The results show this procedure can be used as a method to extract the electrostatic force as a function of the microstructure's degrees of freedom. The procedure can be easily used for any microstructure with a wide variety of electrode configurations to predict the response of the system to any input excitation.
\end{abstract}

Index Terms-MEMS, Dynamics and Vibration, System Identification, Electrostatic Levitation.

\section{INTRODUCTION}

The superior performance and successful implementation of capacitive MEMS transducers such as MEMS microphones [1], accelerometers [2], [3], gyroscopes [4], [5], pressure sensors [6], and micro-mirrors [7] in our everyday lives has motivated researchers to study the multi-physics domain of these tiny devices to improve their performance and to address their shortcomings. This improvement will gradually lead to devices for new applications such as endoscopic imaging, metrology, and energy harvesting [8]-[10].

The most common capacitive transduction scheme in MEMS transducers is to use two electrodes with different voltages that have relative motion with respect to each other [11]. The main drawback of this design is the pull-in instability of the microstructure that happens when the two electrodes get close to each other beyond a threshold gap where the compliant element of the microstructure is no longer able to overcome the electrostatic force [12].

The levitation electrostatic force scheme resolves this issue by omitting the pull-in instability as a microstructure failure mode [13]. In this scheme, there are three fixed electrodes and one moving electrode as shown in Fig. 1 (a). The moving electrode and the fixed electrode beneath it have the same voltage (ground). Applying an electrical voltage on the side fixed electrodes creates an electric field that results in an electrostatic force on the moving electrode. This electrostatic force pushes the moving electrode away from the bottom electrode. This is because the bottom surface of the moving electrode is

M. Daeichin, R. N. Miles, and S. Towfighian are with the Department of Mechanical Engineering, State University of New York at Binghamton, Binghamton, NY, 13902 USA, Corresponding author: S. Towfighian (stowfigh@binghamton.edu) shielded by the bottom electrode and therefore, there are more lines of electric field going to the top surface of the moving electrode compared to the number of lines going to the bottom surface. As a result, the electrostatic force on the top surface, which is upward, is larger than the downward electrostatic force on the bottom surface. This means the net electrostatic force on the moving electrode is upward, pushing it away from the bottom electrode. This nature of the electrostatic force eliminates the possibility of the moving electrode being pulled into the bottom electrode. It also leads to different types of nonlinear behavior distinct from those for transducers based on parallel plate configuration which could be beneficial for some applications. For instance, the parametric resonance of transducers based on this scheme can be exploited to operate micromirrors at higher frequencies while maintaining the large displacement for the mirror [14]. It is worth mentioning that the electrostatic force in the levitation scheme, in general, is smaller than the force in parallel plate scheme because of the configuration of the electrodes. For comprehensive study on the nonlinear dynamics of microstructures based on levitation scheme one could refer to [14]-[16].

In the levitation-based electrode configuration, even if the moving electrode comes into contact with the underlying electrode, there would be no stiction or micro-welding because both electrodes carry the same voltage. This configuration allows for large-amplitude displacement of the moving electrode which is very desirable in applications like micromirrors [14], [17], [18]. It is worth mentioning that similar performance can be achieved with a slightly different design that employs two fixed electrodes and one moving electrode [19]. These unique characteristics of the levitation scheme have inspired researchers to explore its feasibility for a wide range of applications from micro-mirrors and accelerometers to MEMS switches [14], [16], [20], [21]. A comprehensive analysis of the dynamics of the moving electrode in a repulsive electrode configuration is presented in our previous work [15].

To find the electrostatic force on the moving electrode in the levitation scheme, one needs to solve Laplace's equation for the electric field distribution [22]-[25]. For conventional parallel plate or comb drive configurations, the electric field between the electrodes is commonly assumed to be uniform. This practical assumption leads to a simple analytical solution for Laplace's equation [26] and the electrostatic force. However, finding the electric field in the levitation scheme is more challenging. The electric field in this multi-electrode system is not uniform which makes solving Laplace's equation analytically difficult.

The conformal mapping technique [27] can be used to solve 
for an analytical solution of the electric field distribution in a multi-electrode system, but using the analytical result in a mathematical model of the microstructure is cumbersome [28]. In the case of an analytical solution being available, the least squares method can be used to replace the solution with a polynomial form which is mathematically easier to handle.

Finite element and boundary element methods are often used approaches to solve for the electric field in a multielectrode system which is more common than the analytical approach [20], [29]. However, solving the problem in a 3D domain is computationally difficult and inefficient. To circumvent this complexity, the lengths of the electrodes are usually assumed to be infinite so that the problem could be solved in 2D which compromises the accuracy of the solution [29]-[31].

Furthermore, these analytical or finite element solutions are usually achieved for just one unit cell. They do not account for multiple unit cells used next to each other when attached to a larger microstructure. For example, Ozdogan et. al have used multiple arc-shape unit cells attached to a circular micro-mirror plate with 1 diameter [14]. In another work by Daeichin et. al [16] 81 rectangular-shaped unit cells are attached to the 1 by 1 proof-mass plate of an accelerometer. The existence of fabrication imperfections in the microstructure causes the real unit cells to deviate from the simplified 2D model. For instance, in the accelerometer with these unit cells in [16], the proof mass has an initial curvature of $6 \mu \mathrm{m}$ because of the residual stress created during the fabrication process. This curvature affects how the moving electrodes of the levitation units are initially placed above the fixed electrodes and how they move. The difference between the real-world microstructure and the finite element model leads to an inaccurate prediction of the electrostatic force.

In this study, we have proposed a two-step experimental procedure to obtain the levitation electrostatic force in MEMS transducers. The procedure is explained on one microstructure, but it can be used for any microstructure with arbitrary geometry based on levitation electrostatic force. Characterizing the levitation electrostatic force is important for devices that have large surface to thickness ratios and are susceptible to residual stresses and deformation. For these devices, two-dimensional finite element analysis (FEA) does not yield an accurate representation of the electrostatic force, and three-dimensional FEA is computationally expensive. The effectiveness of the proposed approach is examined by using the electrostatic force in a lumped parameter model to predict the frequency response of the microstructure when subjected to a dynamical excitation. The experimentally obtained electrostatic force in this procedure accounts for all the fabrication imperfections and fringing effects of multiple unit cells next to each other for any shape of a levitation unit cell. This procedure is non-invasive and does not lead to any structural or electrical damage to the microstructure.

The working principle and fabrication of the microstructure presented in this study are explained in Section 2. In Section 3 , the experimental procedure and results are presented. In

\begin{tabular}{llrc}
\hline Parameter & Symbol & Value & Unit \\
\hline Proof mass length & $L$ & 1000 & $\mu \mathrm{m}$ \\
Proof mass width & $L$ & 1000 & $\mu \mathrm{m}$ \\
Electrode length & $L_{e}$ & 200 & $\mu \mathrm{m}$ \\
Total number of unit cells & $N$ & 112 & \\
Voltaged fixed electrode width & $d_{1}$ & 4 & $\mu \mathrm{m}$ \\
Gap between fixed electrodes & $d_{2}$ & 6 & $\mu \mathrm{m}$ \\
Moving electrode width & $d_{3}$ & 6 & $\mu \mathrm{m}$ \\
Ground fixed electrode width & $d_{4}$ & 16 & $\mu \mathrm{m}$ \\
Electrodes and Proof thickness & $h_{1}$ & 1.5 & $\mu \mathrm{m}$ \\
Initial gap & $h_{2}$ & 2.75 & $\mu \mathrm{m}$ \\
Mechanical natural frequency & $\omega_{n}$ & $2 \pi \times 1334$ & $\frac{r a d}{S}$ \\
Damping ratio $(V d c=40)$ & $\xi_{0}$ & 0.064 & \\
Damping ratio $(V d c=50)$ & $\xi_{0}$ & 0.071 & \\
Damping ratio $(V d c=60)$ & $\xi_{0}$ & 0.080 & \\
\hline \multicolumn{2}{c}{ TABLE I } & & \\
\multicolumn{2}{c}{ PARAMETERS } & &
\end{tabular}

Section 4, simulation results with the obtained electrostatic force are compared with experimental results for when the microstructure is subjected to different dynamical loads. The conclusion is given in Section 5.

\section{WORKING PRINCIPLE AND FABRICATION}

The schematic of the microstructure considered in this paper is shown in Fig. 1 (c). This microstructure consists of a square plate that is anchored by four supporting springs. The levitation unit cells are employed on all four sides of this plate. When a bias voltage is applied on the side electrodes, the moving electrodes of the levitation unit cells are pushed away from the substrate and lift the microstructure plate up. Depending on the application, this mechanism can be used for sensing and actuation.

For sensing, the microstructure plate would be excited with an external force to vibrate and the change in capacitance between the electrodes is measured. For instance, if used as a MEMS microphone [1], the plate is going to vibrate from an incoming sound pressure wave and the capacitance change is measured. Or, for a MEMS accelerometer [2], the microstructure, when subjected to a base excitation, is going to vibrate because of its inertia. This vibration leads to a change in capacitance that can be measured with an electric circuit [32] and related to base acceleration.

The fabricated microstructure is shown in Fig. 2. The fabrication is done using a POLYMUMPS standard process that allows for three structural polysilicon layers with $0.5 \mu \mathrm{m}$, $2 \mu \mathrm{m}$, and $1.5 \mu \mathrm{m}$ thicknesses and two oxide layers with $2 \mu \mathrm{m}$ and $0.75 \mu \mathrm{m}$ thicknesses from bottom to up respectively.

In the fabricated device, shown in Fig. 2, the three fixed electrodes are made in the first polysilicon layer $(0.5 \mu \mathrm{m}$ thick which is called poly0 in the standard) and then the second polysilicon layer (poly 1 in the standard) is skipped. The microstructure and moving electrodes are fabricated in the third structural layer (poly 2 in the standard with a thickness of $1.5 \mu \mathrm{m})$. Skipping the second layer allows us to stack the two oxide layers on top of each other which when etched, provides a $2.75 \mu \mathrm{m}$ gap between the microstructure and its underlying 


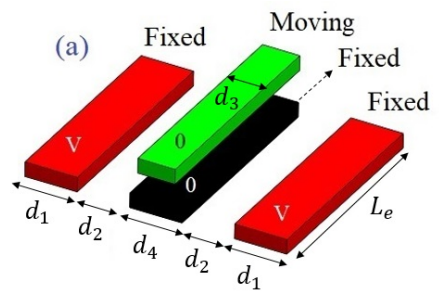

(b)

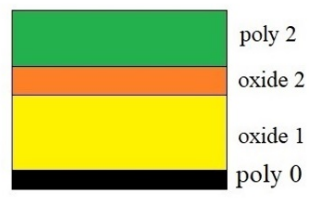

(c)
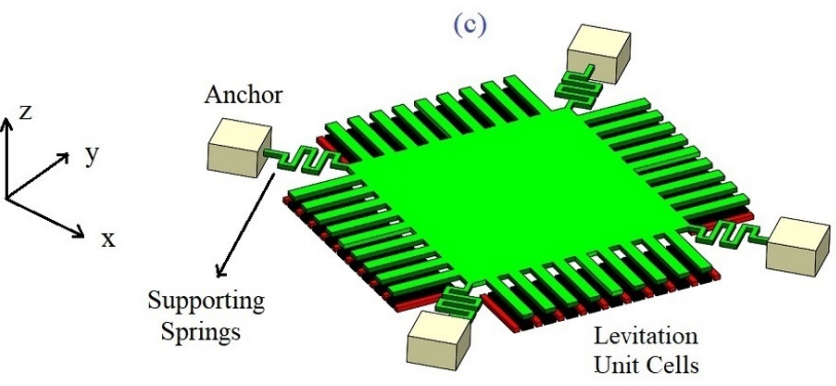

Fig. 1. (a) Repulsive unit cell (not drawn to scale). The dimensions are given in Table I (b) schematic of structural and oxide layers used in the fabrication process, and (c) schematic of the microstructure (not drawn to scale)

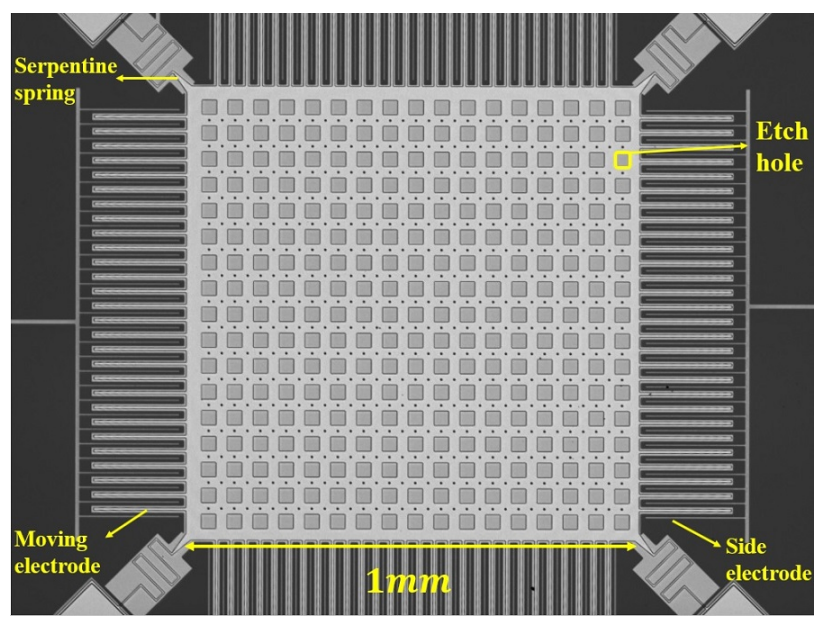

Fig. 2. Top view of the fabricated microstructure. The moving and side electrodes are shown in this figure. The bottom electrodes are underneath the moving electrodes. The microstructure is supported with four serpentine springs. There are several etch holes on the microstructure plate to ensure the release of the microstructure.

substrate (poly0 layer). The schematic of the layers before etching is shown in Fig. 1 (b).

As shown in Fig. 2 there are multiple etch holes equally spaced from each other to ensure the successful releasing of the microstructure during the etching process.

\section{EXPERIMENTAL PROCEDURE AND RESULTS}

This section entails the general procedure to obtain the electrostatic force for the MEMS device shown in Fig 2. This microstructure consists of a 1 by 1 perforated plate which is supported by four serpentine springs at its corners. On each side of the plate, there are 28 sets of levitation unit cells. The degree of freedom (DOF) is considered to be translational in the out-of-plane direction ( $\mathrm{z}$ direction). The goal is to find the electrostatic force on the microstructure as a function of its DOF. There are two measurements that need to be done

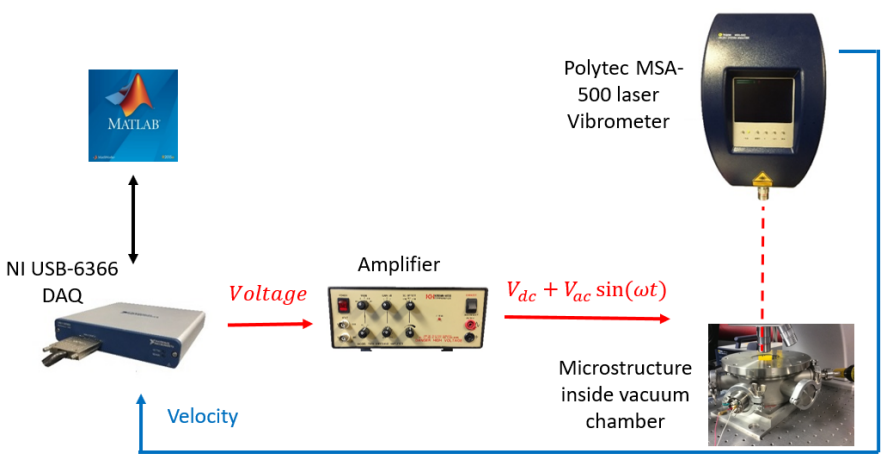

Fig. 3. Experimental setup. The Ac voltage is sent from the NI USB-6366 DAQ to the amplifier. The amplifier amplifies this signal and add the desired DC component to it and then send it to the microstrcuture inside the vacuum chamber. The motion of the microstructure is measured with the polytech MSA-500 laser vibrometer by reading the velocity.

to collect all the data necessary to calculate the electrostatic force normalized with respect to the effective mass of the microstructure. The experimental setup is shown in Fig. 3.

The first measurement is obtained by applying a quasi-static voltage on the side electrodes and recording the displacement with the Polytec MSA-500 laser vibrometer and NI USB 6366 DAQ. A sine function with a duration several orders of magnitudes larger than the microstructure's natural period is chosen for the voltage profile. This large duration of applied load ensures that the microstructure response is quasi-static and therefore the dynamical effects such as viscous energy dissipation and acceleration, which are proportional to the first and second time derivatives of displacement, are negligible. The continuity of the voltage profile and its derivatives with respect to time avoids exerting shock-like excitation on the microstructure. This minimizes the effect of damping or dynamical effects in the displacement of the microstructure. Figure 4 shows the applied voltage and the displacement of the microstructure versus time. Higher voltages lead to larger displacements that result in measuring the electrostatic force for a larger range of DOF. For instance, consider a case where we want to measure the electrostatic force when the microstructure is moving from its initial rest position to 5 microns above this position. In order to have the electrostatic force in this range, we have to apply enough voltage during the experimental procedure to have the microstructure move 5 microns. This means that if we apply higher voltages during the experimental procedure, we can then calculate the electrostatic force for larger range of microstructure displacement. In our calculation, we have considered the first half of the voltage and displacement data where the voltage and displacement go from 0 to their maximum values. According to Fig 4 the displacement at the end of the quasi-static loading (when voltage goes zero) has a small nonzero value. This is because of the inherent drift in laser vibrometer measurement of displacement. If the load time duration is large enough that the drift of the laser over time is significant, then this drift should be removed using a least square method. Here, we have ignored this drift because it is small, especially in the first part 
of the displacement data where displacement goes from 0 to its maximum value (not from maximum to 0 ). Because this experiment is quasi static, the pressure is not important so we have conducted this measurement at atmospheric pressure.

The second measurement is designed to obtain the linearized natural frequency (the resonance frequency at small excitation levels) of the microstructure at different DC voltages on side electrodes. As explained in [14]-[16] the resonance frequency of a microstructure that employs levitation unit cells, increases with the DC voltage on the side electrodes. To measure the linearized natural frequency of the microstructure at a desired DC voltage, first, the DC voltage is applied on the side electrodes. Then, the microstructure is excited slightly while the velocity at one point (here, at the center of the plate) is being recorded with the laser vibrometer. There are three ways to excite the microstructure to get the linearized frequency data.

The first way to excite the microstructure is to apply a small random voltage on the side electrodes (after a DC voltage is initially applied on side electrodes). The frequency at which the magnitude of the transfer function between the random voltage (input) and velocity (output) is maximum yields the linearized natural frequency. The second way is to apply a small AC voltage on top of the initial DC voltage and perform a frequency sweep while measuring the velocity of the device. The peak in the frequency response again yields the linearized natural frequency at the given applied DC voltage. The third way to excite the microstructure is to excite it mechanically with a speaker or a shaker and performing a frequency sweep to obtain the linearized natural frequency. In this study, we have chosen the second way to extract the linearized natural frequencies. It is worth mentioning that all these techniques lead to the same results but the important consideration here is to perturb the microstructure slightly when a DC voltage is applied on the side electrodes. The reason behind this is that the levitation electrostatic units exhibit different nonlinear behaviors like softening and hardening when they undergo large deflections [14]-[16], or parametric and superharmonic resonances when they are excited at half or twice of their fundamental natural frequency [14], [33]. In section 4 , we will use these experimentally obtained resonance frequencies to equate them with linearized natural frequencies obtained from stability analysis using the Jacobian matrix. Linearized natural frequencies represent the microstructure resonance frequencies around their equilibrium state when it is perturbed slightly and this is why it is important to apply small excitation and not provoke any nonlinearities that accompany large excitations and displacements.

It is important to keep track of the pressure in the resonance frequency experiment because the air might introduce stiffness effects to the microstructure. This is because of the squeeze film effect that creates a stiffness and an energy dissipation mode that affects the microstructure dynamics. A comprehensive study of the squeeze film damping effect is given in [26]. It is recommended to perform this test in pressure ranges in which the microstructure is going to be used. Here we have used reduced pressure range of $277-287$ ( $m$ Torr $)$
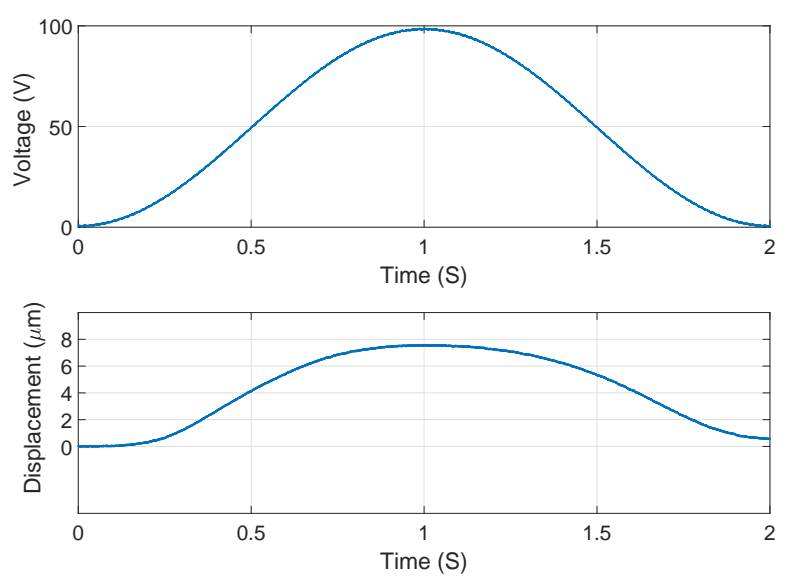

Fig. 4. By applying a quasi-static sinusoidal voltage on the side electrodes, the microstructure undergoes a quasi-static displacement with a similar profile. In this measurement the displacement of the microstructure is recorded with a vibrometer displacement decoder. At the end of the measurement, the displacement is not exactly zero which is because of the drift in the vibrometer reading.

for the natural frequency and all the dynamical experiments. The measured linearized natural frequency at different voltages is shown in Fig. 6. It should be mentioned that, if needed, the effective mass of the microstructure can be calculated by measuring the effective stiffness of the microstructure and using the fundamental natural frequency. The effective stiffness of the microstructure can be measured by using a nano-indenter to apply a mechanical force and recording the microstructure's displacement when there is no voltage on the side electrodes. This procedure is explained in more detail in [34]. Here, because we will use a lumped parameter model that is normalized with respect to effective mass (next section), there is no need for measuring the effective mass.

\section{Mathematical Modeling}

A lumped parameter model with one degree of freedom is considered in Eq. (1) to simulate the microstructure's response at the center of the plate $(x)$.

$$
\ddot{x}+2 \zeta \omega_{n} \dot{x}+\omega_{n}^{2} x=f(x, V)
$$

Where $f(x, V)$ is the electrostatic force normalized with respect to the effective mass of the microstructure, $\zeta$ is the damping ratio, and $\omega_{n}$ is the mechanical natural frequency of the microstructure.

\section{A. Quasi-static motion}

As mentioned before, the quasi-static experiment is done in a way that dynamical effects like acceleration and damping would be negligible. So, by putting the time derivative terms in Eq. (1) equal to zero, the equation governing the static response $\left(x_{s t}\right)$ of the microstructure is obtained (Eq. (2))

$$
\omega_{n}^{2} x_{s t}=f\left(x_{s t}, V_{d c}\right)
$$


This means that as $\omega_{n}$ is known from the resonance frequency measurement, the normalized electrostatic force is known at any equilibrium point $\left(x_{s t}, V_{d c}\right)$.

It is worth mentioning that $f(x, V)$ in Eq. 1 is a scalar function of two variables ( $x$ and $V$ ), and therefore we can define the gradient operator acting on it to calculate its directional derivative along any arbitrary direction in $x-V$ plane. Using this concept, we can calculate the directional derivative of $f(x, V)$ at each equilibrium point along the vector $(\vec{u})$ in $x-V$ plane that is tangent to the $x_{s t}-V_{d c}$ curve as given in Eq. (3). Figure 5 is useful to visualize this directional derivative. Because we have $x_{s t}-V_{d c}$ from the experiment (blue curve in Fig. 5), we can find the tangent line to it at each point.

$d f(x, V)_{\vec{u}}=\vec{\nabla} f(x, V) \cdot \vec{u}=\frac{\partial f\left(x_{s t}, V_{d c}\right)}{\partial x} u_{1}+\frac{\partial f\left(x_{s t}, V_{d c}\right)}{\partial V} u_{2}$

where $\vec{\nabla}$ is the gradient operator and

$$
\vec{u}=u_{1} \vec{i}_{x}+u_{2} \vec{j}_{v}
$$

is the unit vector tangent to $x_{s t}-V_{d c}$ curve at $\left(x_{s t}, V_{d c}\right)$. Unit vectors along $\mathrm{x}$ and $\mathrm{V}$ axis are $\vec{i}_{x}$ and $\vec{j}_{v}$ respectively.

According to Eq. (2), the directional derivative of $f(x, V)$ along the $\left(x_{s t}, V_{d c}\right)$ is:

$$
d f(x, V)_{\vec{u}}=\omega_{n}^{2} \Delta x_{s t}
$$

Therefore, Eq. 3 and Eq. (5) give us:

$$
\frac{\partial f\left(x_{s t}, V_{d c}\right)}{\partial x} u_{1}+\frac{\partial f\left(x_{s t}, V_{d c}\right)}{\partial V} u_{2}=\omega_{n}^{2} \Delta x_{s t}
$$

\section{B. Resonance frequency}

To find the linearized natural frequencies from the model given in Eq. (1), first, we need to write the governing equation in state space. By using state variables $x_{1}$ and $x_{2}$ for displacement $(x)$ and velocity $(\dot{x})$ respectively, the governing equation of motion can be written in state space as described in (Eq. (7)).

$$
\left\{\begin{array}{l}
\dot{x}_{1}=x_{2}=g_{1}\left(x_{1}, x_{2}\right) \\
\dot{x}_{2}=-2 \zeta \omega_{n} x_{2}-\omega_{n}^{2} x_{1}+f\left(x_{1}, V\right)=g_{2}\left(x_{1}, x_{2}\right)
\end{array}\right.
$$

Then, the Jacobian matrix can be constructed as illustrated in (Eq. (8)).

$$
J=\left[\begin{array}{ll}
\frac{\partial g_{1}}{x_{1}} & \frac{\partial g_{1}}{x_{2}} \\
\frac{\partial g_{2}}{x_{1}} & \frac{\partial g_{2}}{x_{2}}
\end{array}\right]
$$

The eigenvalues of the Jacobian matrix for the undamped system yield the linearized natural frequencies at the equilibrium points $\left(x_{1}=x_{s t}\right.$ and $\left.x_{2}=0\right)$. Eq. (9) gives the eigenvalue problem.

$$
|J-\lambda I|=\left|\left[\begin{array}{cc}
-\lambda & 1 \\
-\omega_{n}^{2} x_{s t}+\frac{\partial f\left(x_{s t}, V_{d c}\right)}{\partial x} & -\lambda
\end{array}\right]\right|=0
$$

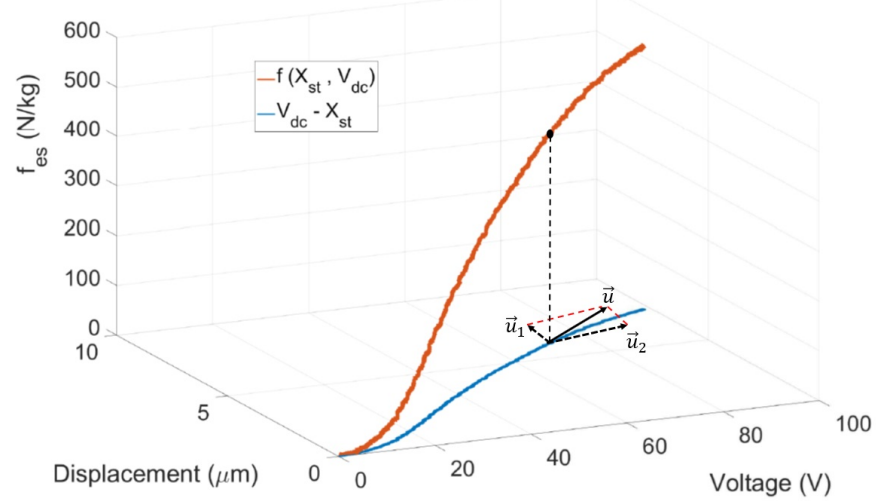

Fig. 5. Electrostatic force and displacement are shown as the voltage on the side electrode varies. The gradient of the electrostatic force is calculated along a unit vector tangent to the voltage-displacement curve in $X-V$ plane.

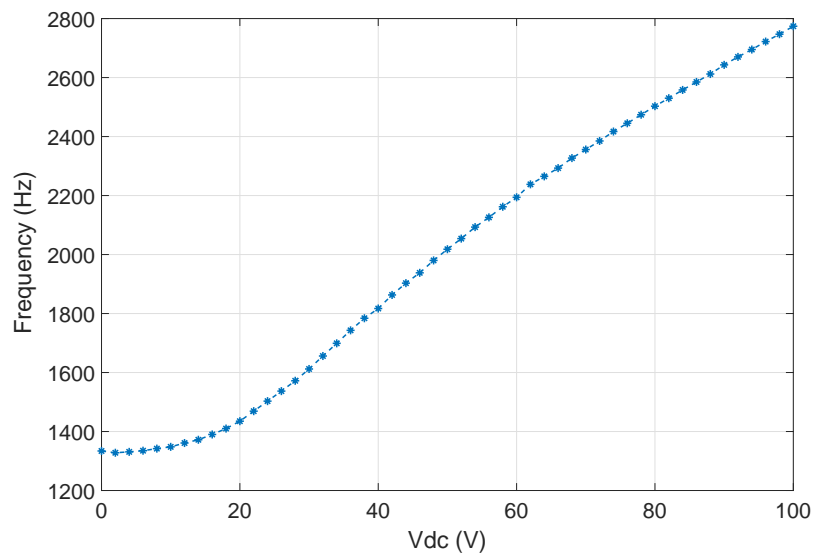

Fig. 6. Resonance frequency for different voltages on the side electrodes is measured. By increasing the voltage on the side electrode, the electrical stiffness increases which lead to an increase in the resonance frequency of the microstructure.

On the other hand, the eigenvalue of the Jacobian matrix $(\lambda)$ is related to the linearized natural frequency through a complex equation given in Eq. (10):

$$
\lambda=i \omega
$$

Where $(\omega)$ is the linearized natural frequency. Solving Eq. (9) for $\lambda$ using Eq. (10) and rearranging the terms gives:

$$
\frac{\partial f\left(x_{s t}, V_{d c}\right)}{\partial x}=\omega_{n}^{2}-\omega^{2}
$$

Equations (6) and (11) construct a linear system of algebraic equations with two equations and two unknowns, $\frac{\partial f\left(x_{s t}, V_{d c}\right)}{\partial x}$ and $\frac{\partial f\left(x_{s t}, V_{d c}\right)}{\partial V}$ that can be easily solved to find the unknowns. After finding these two unknowns, the normalized electrostatic force can be obtained using a first-order Taylor expansion for a two-variable function (Eq. (12)). 


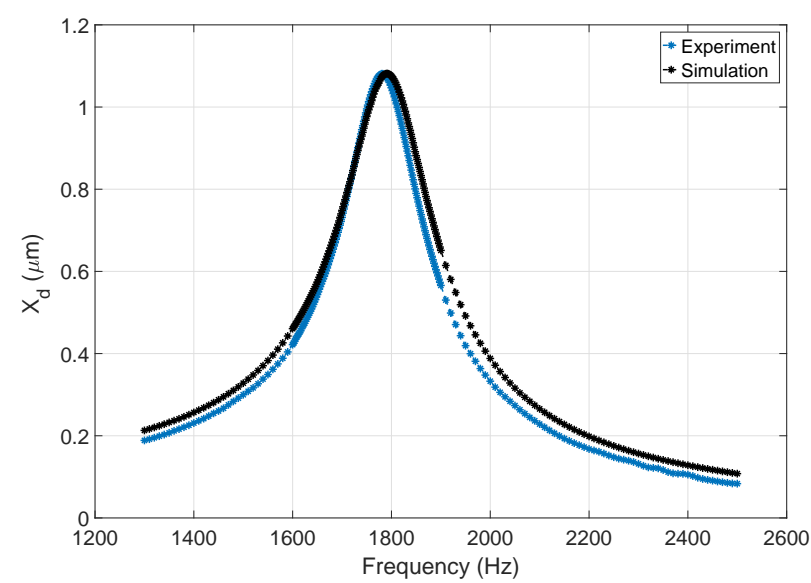

Fig. 7. Dynamical displacement when a time-varying voltage with different frequencies is applied on the side electrodes $\left(V_{d c}=40(V), V_{a c}=1(V)\right)$.

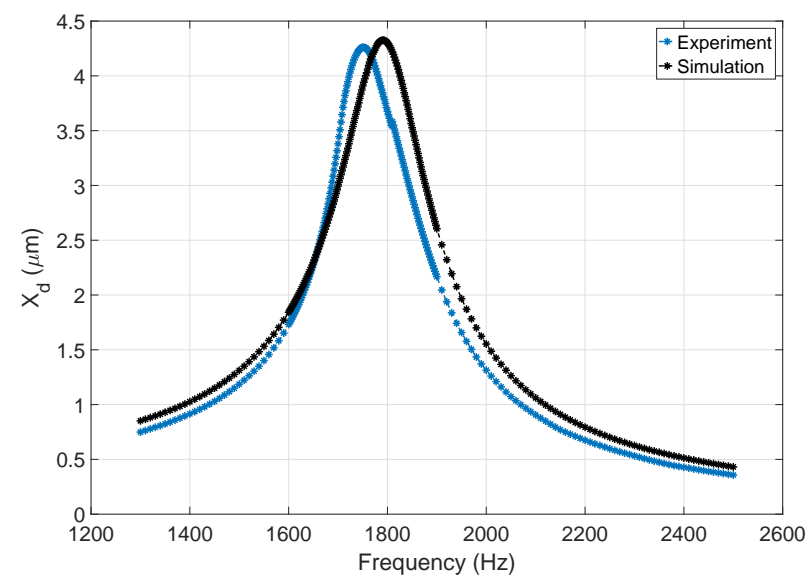

Fig. 8. Dynamical displacement when a time-varying voltage with different frequencies is applied on the side electrodes $\left(V_{d c}=40(V), V_{a c}=4(V)\right)$.

$$
\begin{array}{r}
f(x, V)=f\left(x_{s t}, V_{d c}\right)+\frac{\partial f_{e s}\left(x_{s t}, V_{d c}\right)}{\partial x}\left(x-x_{s t}\right)+ \\
\frac{\partial f_{e s}\left(x_{s t}, V_{d c}\right)}{\partial V}\left(V-V_{d c}\right)+\cdots
\end{array}
$$

This force can be used in the lumped parameter model to predict the microstructure response to dynamical loads. The equation for the lumped parameter model when a $D C+A C$ voltage load is derived using Eq. (1) and Eq. (12).

$$
\begin{aligned}
\ddot{x}+2 \zeta \omega_{n} \dot{x}+\omega_{n}^{2} x=f & \left(x_{s t}, V_{d c}\right)+\frac{\partial f\left(x_{s t}, V_{d c}\right)}{\partial x}\left(x-x_{s t}\right)+ \\
& \frac{\partial f\left(x_{s t}, V_{d c}\right)}{\partial V}\left(V-V_{d c}\right)+\cdots \quad(13)
\end{aligned}
$$

The $D C+A C$ voltage that is applied to the side electrodes can be written as:

$$
V=V_{d c}+V_{a c} \sin \left(\omega_{e} t\right)
$$

Where $V_{d c}$ and $V_{a c}$ are the DC and AC voltage amplitudes respectively and $\omega_{e}$ is the excitation frequency. The

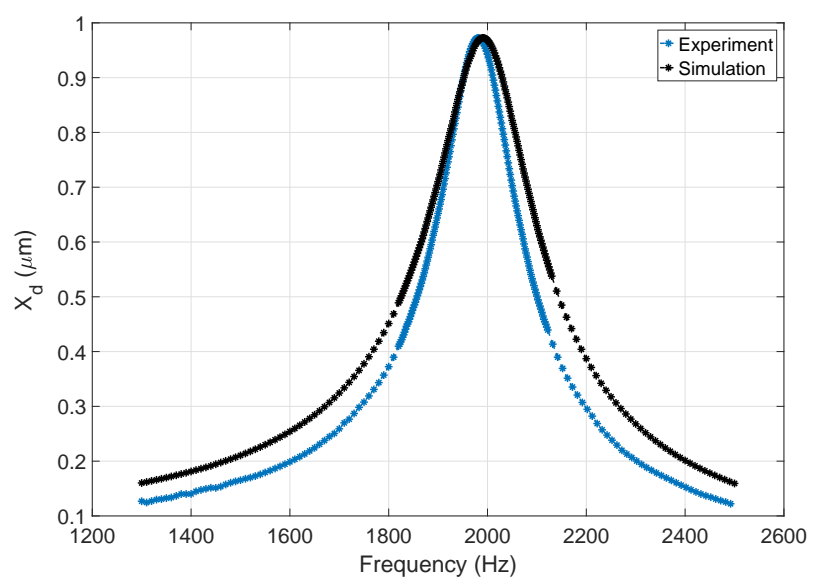

Fig. 9. Dynamical displacement when a time-varying voltage with different frequencies is applied to the side electrodes $\left(V_{d c}=50(V), V_{a c}=1(V)\right)$.

microstructure response to this load can be split in to two parts, a static part $\left(x_{s t}\right)$ which is caused by $V_{d c}$ and a dynamic part $\left(x_{d}\right)$ which is from $V_{a c} \sin \left(\omega_{e} t\right)$, i.e.

$$
x=x_{s t}+x_{d}
$$

Where $x_{s t}$ is picked from the quasi-static experiment for the corresponding $V_{d c}$. Substituting this change of variable into Eq. (13) yields:

$$
\begin{aligned}
& \ddot{x_{d}}+ 2 \zeta \omega_{n} \dot{x_{d}}+\omega_{n}^{2}\left(x_{s t}+x_{d}\right)= \\
& f\left(x_{s t}, V_{d c}\right)+\frac{\partial f\left(x_{s t}, V_{d c}\right)}{\partial x}\left(x_{s t}+x_{d}-x_{s t}\right)+ \\
& \frac{\partial f\left(x_{s t}, V_{d c}\right)}{\partial V}\left(V_{d c}+V_{a c} \sin \left(\omega_{e} T t\right)-V_{d c}\right)+\cdots
\end{aligned}
$$

Using Eq. (2) and some mathematical simplification and assuming that the higher-order terms in the Taylor series expansion are small for small AC excitations, Eq. (16) can be rewritten in the following form:

$$
\begin{aligned}
\ddot{x_{d}}+2 \zeta \omega_{n} \dot{x_{d}}+\omega_{n}^{2} x_{d}= & \frac{\partial f\left(x_{s t}, V_{d c}\right)}{\partial x} x_{d}+ \\
& \frac{\partial f\left(x_{s t}, V_{d c}\right)}{\partial V} V_{a c} \sin \left(\omega_{e} T t\right)
\end{aligned}
$$

The coefficients on the right-hand side are now identified, and Eq. (17) can be solved for different load cases $\left(V_{d c}\right.$ and $\left.V_{a c}\right)$ to find the dynamical displacement of the microstructure $\left(x_{d}\right)$. All the other parameters in this equation are known except for the damping ratio which is identified from comparing the resonance peaks at different DC voltages given in Table I. The dependency of the damping ratio to the voltage on side electrodes is explained in [14], [16]. For a comprehensive review of quality factor tuning in MEMS resonators, one can refer to [35]. Equation (17) can be solved using the shooting method explained in [26]. All the simulated frequency responses in the paper are obtained by solving this equation using the shooting method. 


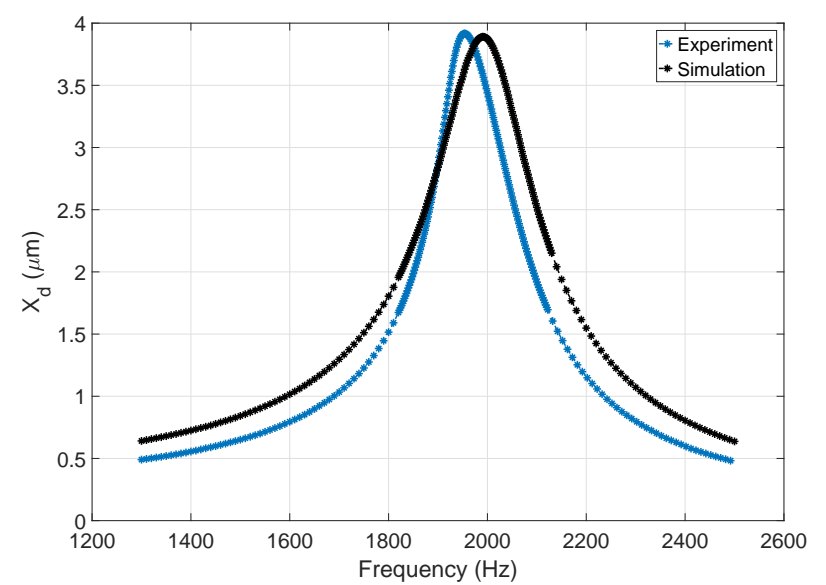

Fig. 10. Dynamical displacement when a time-varying voltage with different frequencies is applied to the side electrodes $\left(V_{d c}=50(V), V_{a c}=4(V)\right)$.

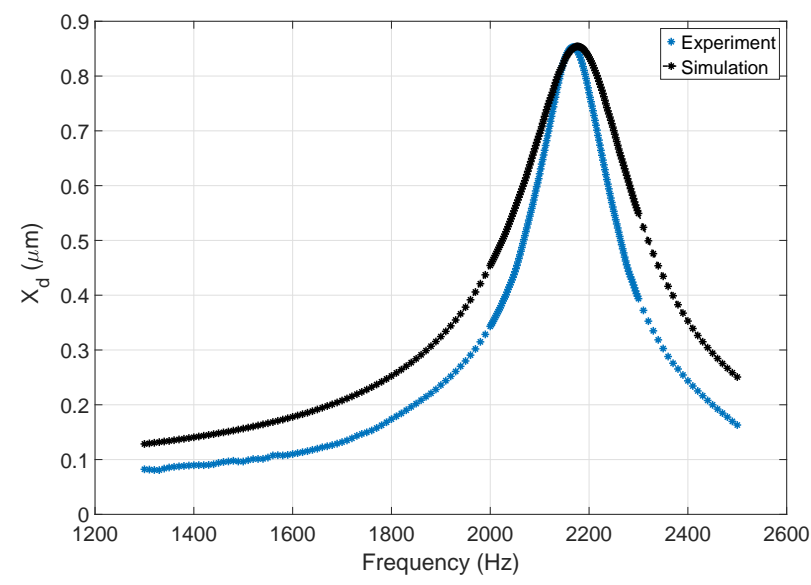

Fig. 11. Dynamical displacement when a time-varying voltage with different frequencies is applied to the side electrodes $\left(V_{d c}=60(V), V_{a c}=1(V)\right.$ ).

Because the first-order Taylor expansion is used to obtain the electrostatic force, the force and the model should give better results for small excitations compared to large excitations. Figures 7, 8, 9, 10, 11, 12 show the lumped model results compared to experimental results for different voltage excitation. A summary of the results is given in Table II. In this table, the bandwidth is considered to be the width of the frequency range where the dynamical amplitude is larger than $\frac{1}{\sqrt{2}}$ of the resonance amplitude peak. Comparing Figs. 7, 9, and 11 reveals the increase in the resonance frequency by an increase in the voltage on the side electrodes. For example, the increase of DC excitation voltage to $50(\mathrm{~V})$ from $40(\mathrm{~V})$ shifts the resonant frequency to $1990(\mathrm{~Hz})$ from $1790(\mathrm{~Hz})$. An increase of $\mathrm{AC}$ voltage at the same $\mathrm{DC}$ level causes an increase of dynamic amplitude, compare Figs. 7 and 8, Figs. 9 and 10, and Figs. 11 and 12. However, an increase of DC voltage at the same AC amplitude leads to a decrease of dynamic amplitude (Figs. 7, 9, 11 and Figs. 8, 10, 12) because of the increase of electrical stiffness as depicted in Fig. 6. These two properties of levitation transducers are

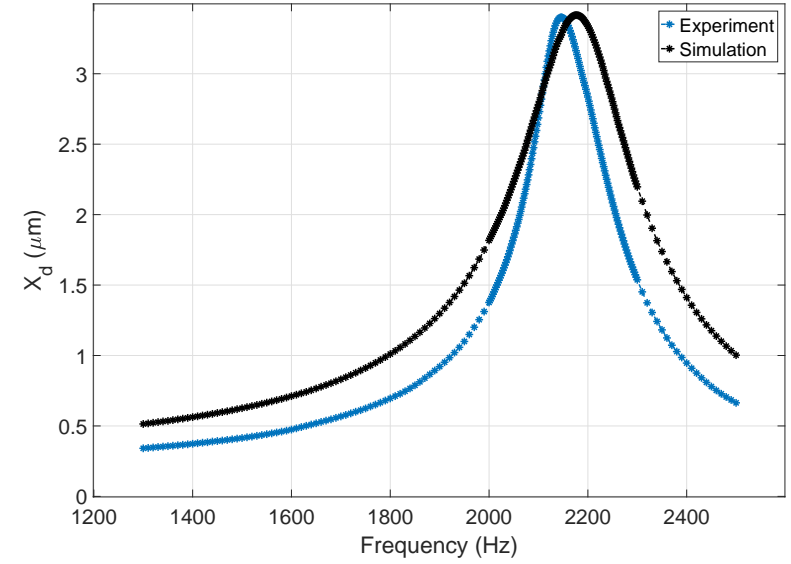

Fig. 12. Dynamical displacement when a time-varying voltage with different frequencies is applied to the side electrodes $\left(V_{d c}=60(V), V_{a c}=4(V)\right)$.

in contrast to parallel-plate actuators that show decrease of electrical stiffness as the DC voltage increases.

It is worth mentioning that at $V d c=40$ the static displacement of the microstructure is about $2 \mu \mathrm{m}$. This displacement will add to the initial gap between microstructure and the substrate $(2.75 \mu \mathrm{m})$ to provide $4.75 \mu \mathrm{m}$ of room in total for the vibration of the microstructure. Figure 8 shows that the maximum amplitude of the microstructure at its resonance peak is about $4.3 \mu \mathrm{m}$, which means the microstructure is vibrating close to its limit of hitting the substrate. Therefore, even though we obtained the electrostatic force with the small excitation assumption, we can see that the experimentally obtained electrostatic force delivers a very good approximation for when the dynamical amplitude of the microstructure is more than twice as of the static displacement and the microstructure is vibrating close to its physical limits. However, the slight softening observed in the experimental data could not be captured by the model because of the linear expansion for the electrostatic force (first-order Taylor expansion).

\section{Conclusion}

In this study, an experimental procedure to obtain the electrostatic levitation force in MEMS transducers with levitation unit cell configurations is presented. The accuracy of the obtained electrostatic force is examined by using it in a lumped parameter model and solving this model for the frequency response of the microstructure to different dynamical loads. A microstructure is fabricated using the POLYMUMPs standard and it is tested with the aid of a laser vibrometer. The simulation and experimental results are compared with each other which shows a close agreement. The effectiveness of the model to capture the dynamical response of the microstructure demonstrates the effectiveness of the proposed procedure to identify the electrostatic force. The proposed method can be employed for non-traditional electrode systems, whose response can deviate from analytical or numerical approaches because of fabrication imperfections and tolerances. The identified electrostatic force from two simplified tests can be used 


\begin{tabular}{|l|c|c|c|c|c|c|c|c|c|}
\hline \multirow{2}{*}{ Load case } & \multicolumn{3}{|c|}{ Peak Frequency (Hz) } & \multicolumn{3}{|c|}{ Peak amplitude $(\mu \mathrm{m})$} & \multicolumn{3}{c|}{ Bandwidth (Hz) } \\
\cline { 2 - 11 } & Exp & Sim & Error & Exp & Sim & Error & Exp & Sim & Error \\
\hline$V_{d c}=40-V_{a c}=1$ & 1780 & 1790 & $0.56 \%$ & 1.08 & 1.08 & 0 & 150 & 173 & $15 \%$ \\
\hline$V_{d c}=40-V_{a c}=2$ & 1776 & 1792 & $0.9 \%$ & 2.16 & 2.16 & 0 & 148 & 170 & $8 \%$ \\
\hline$V_{d c}=40-V_{a c}=3$ & 1764 & 1792 & $1.59 \%$ & 3.25 & 3.24 & $-0.28 \%$ & 147 & 170 & $16 \%$ \\
\hline$V_{d c}=40-V_{a c}=4$ & 1752 & 1790 & $2.2 \%$ & 4.26 & 4.33 & $1.5 \%$ & 156 & 170 & $9 \%$ \\
\hline$V_{d c}=50-V_{a c}=1$ & 1982 & 1990 & $0.4 \%$ & 0.97 & 0.97 & 0 & 144 & 190 & $32 \%$ \\
\hline$V_{d c}=50-V_{a c}=2$ & 1976 & 1990 & $0.7 \%$ & 1.94 & 1.95 & $0.5 \%$ & 146 & 193 & $32 \%$ \\
\hline$V_{d c}=50-V_{a c}=3$ & 1968 & 1990 & $1.12 \%$ & 2.92 & 2.92 & 0 & 145 & 191 & $32 \%$ \\
\hline$V_{d c}=50-V_{a c}=4$ & 1954 & 1990 & $1.8 \%$ & 3.92 & 3.89 & $0.8 \%$ & 144 & 191 & $33 \%$ \\
\hline$V_{d c}=60-V_{a c}=1$ & 2168 & 2176 & $0.37 \%$ & 0.85 & 0.85 & 0 & 144 & 216 & $50 \%$ \\
\hline$V_{d c}=60-V_{a c}=2$ & 2162 & 2176 & $0.65 \%$ & 1.74 & 1.71 & $-1.7 \%$ & 139 & 212 & $52 \%$ \\
\hline$V_{d c}=60-V_{a c}=3$ & 2156 & 2176 & $0.93 \%$ & 2.55 & 2.56 & $0.4 \%$ & 142 & 213 & $50 \%$ \\
\hline$V_{d c}=60-V_{a c}=4$ & 2146 & 2176 & $1.4 \%$ & 3.40 & 3.42 & $0.6 \%$ & 142 & 212 & $49 \%$ \\
\hline
\end{tabular}

COMPARING EXPERIMENTAL AND SIMULATION RESULTS FOR DIFFERENT LOAD CASES. THE PEAK AMPLITUDES ARE COMPARED TO THE ONE HUNDREDTH OF A MICROMETER.

to study the performance of microstructures such as sensors or actuators.

\section{ACKNOWLEDGMENT}

The authors would like to acknowledge the financial support of this study by the National Science Foundation (NSF) through grant ECCS 1608692.

\section{REFERENCES}

[1] R. N. Miles, W. Cui, Q. T. Su, and D. Homentcovschi, "A mems low-noise sound pressure gradient microphone with capacitive sensing," Journal of Microelectromechanical Systems, vol. 24, no. 1, pp. 241-248, 2015.

[2] M. G. Guney, X. Li, V. P. J. Chung, J. Paramesh, T. Mukherjee, and G. K. Fedder, "High dynamic range cmos-mems capacitive accelerometer array," 2018 IEEE Micro Electro Mechanical Systems (MEMS), pp. 992 995, Jan 2018.

[3] R. Mukhiya, P. Agarwal, S. Badjatya, M. Garg, P. Gaikwad, S. Sinha, A. K. Singh, and R. Gopal, "Design, modelling and system level simulations of drie-based mems differential capacitive accelerometer,' Microsystem Technologies, Jan 2019.

[4] C. Yang, S. Tang, and N. Tavassolian, "Utilizing gyroscopes towards the automatic annotation of seismocardiograms," IEEE Sensors Journal, vol. 17, no. 7, pp. 2129-2136, April 2017.

[5] A. Sheikhaleh, K. Jafari, and K. Abedi, "Design and analysis of a novel moems gyroscope using an electrostatic comb-drive actuator and an optical sensing system," IEEE Sensors Journal, vol. 19, no. 1, pp. 144 150, April 2019.

[6] F. M. A. Mohammad H Hasan and H. M. Ouakad, "Novel threshold pressure sensors based on nonlinear dynamics of mems resonators," Journal of Micromechanics and Microengineering, vol. 28, no. 6, 2018.

[7] K. B. Lee and Y.-H. Cho, "Amplitude- and gas pressure-dependent nonlinear damping of high-q oscillatory mems micro mirrors," Journal of Microelectromechanical Systems, vol. 27, no. 3, pp. 383-391, June 2018.

[8] H. Li, X. Duan, and T. D. Wang, "An electrostatic mems scanner with inplane and out-of-plane two-dimensional scanning capability for confocal endoscopic in vivo imaging," 2017 IEEE 30th International Conference on Micro Electro Mechanical Systems (MEMS), pp. 514-517, 2017.

[9] J. J. L. K. B. Alexander Stange, Matthias Imboden and D. J. Bishop, "Building a casimir metrology platform with a commercial mems sensor," Microsystems \& Nanoengineering, vol. 5, 2019.

[10] Z. Saadatnia, E. Esmailzadeh, and H. E. Naguib, "High performance triboelectric nanogenerator by hot embossing on self-assembled microparticles," Advanced Engineering Materials, vol. 21, no. 1, 2019.

[11] Z. Mohammed, I. M. Elfadel, and M. Rasras, "High dynamic range zaxis hybrid spring mems capacitive accelerometer," in 2018 Symposium on Design, Test, Integration Packaging of MEMS and MOEMS (DTIP), May 2018, pp. 1-4.
[12] A. H. Nayfeh, M. I. Younis, and E. M. Abdel-Rahman, "Dynamic pull-in phenomenon in mems resonators," Nonlinear Dynamics, vol. 48, no. 1, pp. 153-163, Apr 2007.

[13] W. M. C. Q. Z. C. Weimin Wang, Hao Ren and B. Fan, "Electrostatic repulsive out-of-plane actuator using conductive substrate," Scientific Reports, 2016.

[14] M. Ozdogan, M. Daeichin, A. Ramini, and S. Towfighian, "Parametric resonance of a repulsive force mems electrostatic mirror," Sensors and Actuators A: Physical, vol. 265, 2017.

[15] M. Pallay, M. Daeichin, and S. Towfighian, "Dynamic behavior of an electrostatic mems resonator with repulsive actuation," Nonlinear Dynamics, vol. 89, no. 2, 2017.

[16] M. Daeichin, M. Ozdogan, S. Towfighian, and R. N. Miles, "Dynamic response of a tunable mems accelerometer based on repulsive force," Sensors \& Actuators: A. Physical, 2019.

[17] H. Li, P. Barnes, E. Harding, X. Duan, T. D. Wang, and K. R. Oldham, "Large-displacement vertical electrostatic microactuator dynamics using duty-cycled softening/stiffening parametric resonance," Journal of Microelectromechanical Systems, pp. 1-11, 2019.

[18] S. Towfighian, A. Seleim, E. Abdel-Rahman, and G. Heppler, "A largestroke electrostatic micro-actuator," Journal of Micromechanics and Microengineering, vol. 21, no. 7, 2011.

[19] K. B. Lee and Y.-H. Cho, "Laterally driven electrostatic repulsiveforce microactuators using asymmetric field distribution," Journal of Microelectromechanical Systems, Feb 2001.

[20] M. Pallay and S. Towfighian, "A reliable mems switch using electrostatic levitation," Applied Physics Letter, 2018.

[21] M. Pallay, A. I. Ibrahim, R. N. Miles, and S. Towfighian, "Pairing electrostatic levitation with triboelectric transduction for high-performance self-powered mems sensors and actuators," Applied Physics Letters, vol. 115, no. 13, p. 133503, 2019.

[22] H. M. Ouakad, "Structural behavior of microbeams actuated by outof-plane electrostatic ftinging-fields," Proceedings of the ASME 2013 International Mechanical Engineering Congress and Exposition, San Diego, California, USA, 2013.

[23] H. M. Ouakad, "Numerical model for the calculation of the electrostatic force innon-parallel electrodes for mems applications," Journal of Electrostatics, vol. 76, pp. 254-261, 2015.

[24] H. M. Ouakad and H. M. Sedighi, "Static response and rree vibration of mems arches assuming out-of-plane actuation pattern," International Journal of Non-Linear Mechanics, vol. 110, pp. 44-57, 2019.

[25] H. M. Ouakad and F. Najar, "Nonlinear dynamics of mems arches assuming out-of-plane actuation arrangement," Journal of Vibration and Acoustics, vol. 141, no. 4, 2019.

[26] M. I. Younis, MEMS Linear and Nonlinear Statics and Dynamics. Wiley, New York, 2008.

[27] D. Hah, "Analytical design of linear variable capacitors with shapedfinger comb-drive actuators," 2018 Symposium on Design, Test, Integration Packaging of MEMS and MOEMS (DTIP), pp. 1-5, May 2018.

[28] S. He and R. Ben Mrad, "Large-stroke microelectrostatic actuators for vertical translation of micromirrors used in adaptive optics," IEEE Transactions on Industrial Electronics, vol. 52, no. 4, 2005. 
[29] B. R. I. Slava Krylov and S. Lulinsky, "Bistability of curved microbeams actuated by fringing electrostatic fields," Nonlinear Dynamics, vol. 66, p. $403-426,2011$.

[30] S. Krylov, N. Molinazzi, T. Shmilovich, U. Pomerantz, and S. Lulinsky, "Parametric excitation of flexural vibrations of micro beams by fringing electrostatic fields," Proceedings of the ASME 2010 International Design Engineering Technical Conferences and Computers and Information in Engineering Conference, IDETC-CIE 2010, Montreal, Quebec, Canada.

[31] S. L. Yoav Linzon, Bojan Ilic and S. Krylov, "Efficient parametric excitation of silicon-on-insulator microcantilever beams by fringing electrostatic fields," Journal of Applied Physics, vol. 113, 2013.

[32] R. N. Miles, "A compliant capacitive sensor for acoustics: Avoiding electrostatic forces at high bias voltages," IEEE Sensors Journal, vol. 18, no. 14, pp. 5691-5698, July 2018.

[33] C. D. Botello M, Beatriz J, "Voltage response of circular plate mems resonators under superharmonic resonance," ASME. ASME International Mechanical Engineering Congress and Exposition, vol. 4B: Dynamics, Vibration, and Contro, 2018.

[34] M. I. Younis, F. M. Alsaleem, R. Miles, and Q. Su, "Characterization of the performance of capacitive switches activated by mechanical shock," Journal of Micromechanics and Microengineering, vol. 17, no. 7, June 2007.

[35] J. M. L. Miller, A. Ansari, D. B. Heinz, Y. Chen, I. B. Flader, D. D. Shin, L. G. Villanueva, and T. W. Kenny, "Effective quality factor tuning mechanisms in micromechanical resonators," Applied Physics Reviews, vol. 5, no. 4, p. 041307, 2018.

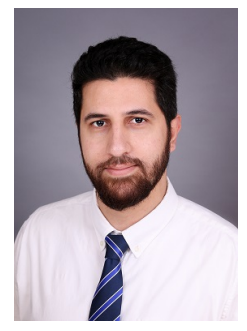

Meysam Daeichin Meysam Daeichin is a $\mathrm{PhD}$ candidate at Binghamton University. Meysam has received his MS degree in Mechanical Engineering from Sharif University of Technology at Tehran, Iran in 2013. He joined the lab in September 2015. He is working towards his $\mathrm{PhD}$ and his research is focused on dynamics and vibration of MEMS transducers.

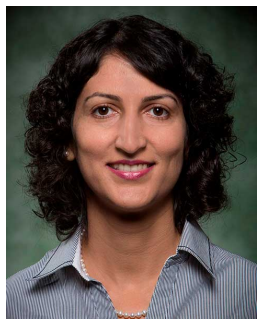

Shahrzad Towfighian Dr. Towfighian received her $\mathrm{PhD}$ degree from the University of Waterloo, Canada in 2011. She joined the Mechanical Engineering department at Binghamton University in Fall 2013. She has published about 40 international journal and conference papers. She has been the recipient of several grants from National Science Foundation and National Health Institute. Her research interests are Micro-electro-mechanical systems and energy harvesting for biomedical devices. She focuses on creating theoretical and experimental frameworks to explain the underlying mechanism of electromechanical systems. Using these frameworks, she seeks innovative methods to improve functionality of devices for various applications.

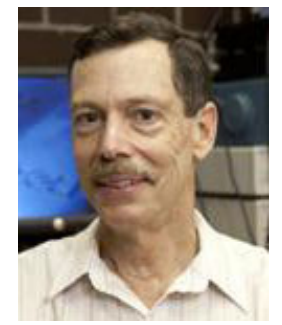

Ronald Miles received the B.S. degree in electrical engineering from the University of California at Berkeley, Berkeley, CA, USA, and the M.S. and $\mathrm{Ph} . \mathrm{D}$. degrees in mechanical engineering from the University of Washington. He has been with the Department of Mechanical Engineering, SUNY Binghamton, Binghamton, NY, USA, since 1989. He has served as the Director of Graduate Studies, the Director of Undergraduate Studies, Associate Chair, Professor, Distinguished Professor, Associate Dean for Research, and currently serves as the Chair of the Department of Mechanical Engineering. 